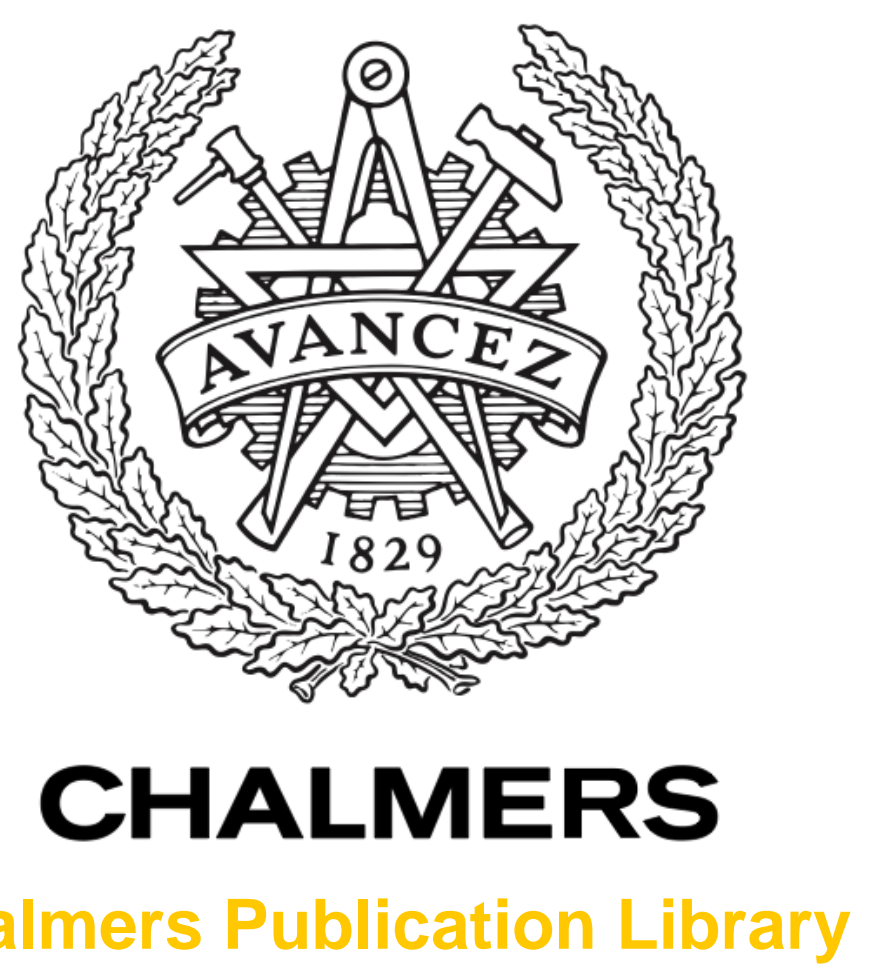

\title{
Wave propagators for the Timoshenko beam
}

This document has been downloaded from Chalmers Publication Library (CPL). It is the author's version of a work that was accepted for publication in:

Wave motion (ISSN: 0165-2125)

Citation for the published paper:

Billger, D. ; Folkow, P. (2003) "Wave propagators for the Timoshenko beam". Wave motion, vol. 37(4), pp. 313-332.

\section{http://dx.doi.org/10.1016/S0165-2125(02)00094-X}

Downloaded from: http://publications.lib.chalmers.se/publication/170244

Notice: Changes introduced as a result of publishing processes such as copy-editing and formatting may not be reflected in this document. For a definitive version of this work, please refer to the published source. Please note that access to the published version might require a subscription. 


\title{
Wave propagators for the Timoshenko beam
}

\author{
Dag V.J. Billger ${ }^{a}$, Peter D. Folkow ${ }^{b, *}$ \\ ${ }^{a}$ The Imego Institute, Aschebergsgatan 46, S-411 33 Göteborg, Sweden \\ ${ }^{b}$ Department of Mechanics, Chalmers University of Technology, S-412 96 Göteborg, Sweden
}

\begin{abstract}
The propagation and scattering of waves on the Timoshenko beam are investigated by using the method of wave propagators. This method is more general than the scattering operators connected to the imbedding and Green function approaches; the wave propagators map the incoming field at an internal position onto the scattering fields at any other internal position of the scattering region. This formalism contains the imbedding method and Green function approach as special cases. Equations for the propagator kernels are derived, as are the conditions for their discontinuities. Symmetry requirements on certain coupling matrices originating from the wave splitting are considered. They are illustrated by two specific examples. The first being an unrestrained beam with a varying cross section and the other a homogeneous, viscoelastically restrained beam. A numerical algorithm for solving the equations for the propagator kernels is described. The algorithm is tested for the case of a viscoelastically restrained, homogeneous beam. In a limit these results agree with the ones obtained for the reflection kernel by a previously developed algorithm for the imbedding reflection equation.
\end{abstract}

\section{Introduction}

In the recent past, several direct and inverse wave propagation problems for the Timoshenko beam have been addressed using time domain techniques. The start of such analysis in beam theory being marked by the discovery of the wave splitting of the Timoshenko beam equation by Olsson and Kristensson [1]. The wave splitting was used together with the Green function technique in [2] for the direct propagation of waves on a free, homogeneous beam. In [3] the imbedding method was

\footnotetext{
${ }^{*}$ E-mail address:peter.folkow@me.chalmers.se
} 
applied to derive equations for the reflection and transmission operator kernels. The imbedding equations have subsequently been used in the investigation of direct problems $[4,5]$ and have also found application in inverse beam problems such as the reconstruction of the varying cross section of a free inhomogeneous beam, see [4]. Another inverse problem is treated in [6] and deals with the reconstruction of the layer properties of a homogeneous beam on a semi-infinite viscoelastic foundation.

The present paper concerns the propagation of flexural waves on an inhomogeneous and viscoelastically restrained Timoshenko beam, utilizing the propagator technique and the wave splitting of a free homogeneous beam [1]. The wave splitting results in coupled equations for the split fields in the inhomogeneous and restrained region [3]. The various fields in this region are related through propagators, which are operators mapping the incoming field at a certain cross section onto the scattered fields at an arbitrary position. The propagator technique was in this context introduced by Karlsson and Stewart [7] for dispersive electromagnetic wave propagation. Since then, the method has been applied extensively to electromagnetic problems $[8,9,10,11,12,13,14]$, but also to elastodynamic [15] and viscoelastic [16] wave propagation problems.

The properties of the propagating waves for small wave front times are of considerable interest in electrodynamics $[8,9,13,14,17,18]$. Here the early time behavior of the propagators, characterized by rapid oscillations and high amplitudes, is approximated by time-domain precursors using various power series expansions. These effects are not seen in the present paper which make the precursor effects negligible. More accurate approximations are obtained by omitting convolutional terms in the propagator expressions. However, since the solution of the propagator equation is not very time consuming, no results based on various approximated equations are presented in this paper. A way of estimating the contributions from precursors are given in [9].

Section 2 briefly reviews the equations of an inhomogeneous Timoshenko beam on a viscoelastic support. The concepts of wave splitting and travel time coordinate transformation are summarized in Section 3. Section 4 introduces the wave propagator formalism and states explicit representations for the propagators in terms of integral kernels. The equations for the propagator kernels are derived in Section 5 and the corresponding discontinuities are treated in Section 6 . The relation of the wave propagators to the imbedding technique and the Green function approach is given in Section 7. Section 8 discusses symmetries of the coupling matrices and their representations for two different cases. The numerical algorithm when solving for the propagator kernels is presented in Section 9 and some numerical examples are given in Section 10 for a homogeneous 
beam on a viscoelastic support. Finally, the propagator kernel equations and the corresponding discontinuities in the case when the sources are located to the right of the scattering region are presented and discussed in Appendix A.

\section{Preliminary remarks}

In this and the next section, some of the basic work presented in [3] is briefly reviewed. Consider a beam which is inhomogeneous and vertically restrained over a finite region (Figure 1). This part of the beam will henceforth be referred to as the region of inhomogeneity and its length is taken to be $d$. The restraint is modelled by uniformly distributed, locally acting springs that include the effect of viscoelastic damping. The springs act on the displacement and the rotation of a beam section.

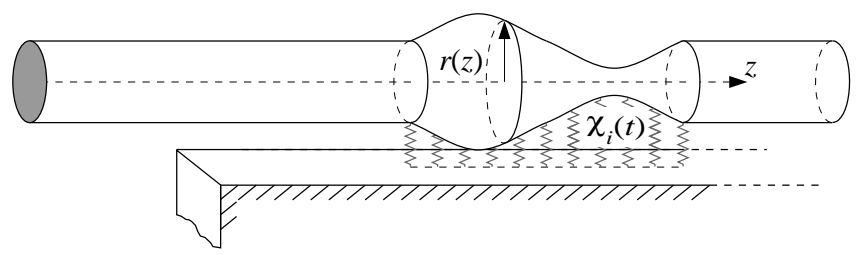

Figure 1: Inhomogeneous beam on viscoelastic suspension.

Using the Timoshenko beam theory [19], the equations of motion are written

$$
\begin{aligned}
& \frac{\partial}{\partial z}\left(f_{1} \gamma\right)-k_{1} u-K_{1} * u=\rho A \frac{\partial^{2} u}{\partial t^{2}} \\
& \frac{\partial}{\partial z}\left(f_{2} \frac{\partial \psi}{\partial z}\right)+f_{1} \gamma-k_{2} \psi-K_{2} * \psi=\rho I \frac{\partial^{2} \psi}{\partial t^{2}}
\end{aligned}
$$

where $u(z, t), \psi(z, t)$ and $\gamma(z, t)$ are the mean transverse deflection, the mean rotation and the mean shear angle of the cross section, respectively [20]. Here, $A$ and $I$ are the area and the moment of inertia of the cross section, while $\rho$ is the density of the beam. The shear stiffness is given by $f_{1}=k^{\prime} G A$ and the bending stiffness is denoted $f_{2}=E I$, where $E$ is the modulus of elasticity and $G$ is the shear modulus. The shear coefficient $k^{\prime}$ depends on the dimensions of the cross section and on Poisson's ratio $\nu[20]$. Note that $A, I, \rho, E, G$ and $k^{\prime}$ may all vary along the beam inside the region of inhomogeneity. For convenience, the $z$-dependence is suppressed in these and the following expressions. The main contribution to the elastic part of the external forces is modelled by spring constants $k_{i}$. The viscous behaviour is modelled by convolutions with memory functions $K_{i}(t)$. Hence, the response of the suspension is influenced by the time history of mean deflection 
and mean rotation. Note that the memory functions also contribute to the elastic part of the response.

Using the relation $\partial_{z} u=\psi+\gamma,(2.1)$ can be given as a system of equations

$$
\partial_{z}\left(\begin{array}{c}
u \\
\psi \\
\gamma \\
\partial_{z} \psi
\end{array}\right)=\left(\begin{array}{cccc}
0 & 1 & 1 & 0 \\
0 & 0 & 0 & 1 \\
c_{1}^{-2} \partial_{t}^{2}+\chi_{1} & 0 & -\partial_{z} \ln f_{1} & 0 \\
0 & c_{2}^{-2} \partial_{t}^{2}+\chi_{2} & -f_{1} / f_{2} & -\partial_{z} \ln f_{2}
\end{array}\right)\left(\begin{array}{c}
u \\
\psi \\
\gamma \\
\partial_{z} \psi
\end{array}\right)
$$

where the two velocities $c_{1}=\sqrt{k^{\prime} G / \rho}$ (effective shear velocity) and $c_{2}=\sqrt{E \rho}$ (rod velocity) satisfy the inequality $c_{1}<c_{2}$. The operators $\chi_{1}$ and $\chi_{2}$ are defined as $\chi_{1}=\left(k_{1}+K_{1} *\right) / f_{1}$ and $\chi_{2}=\left(k_{2}+K_{2} *\right) / f_{2}$. The choice of dependent variables $\left\{u, \psi, \gamma, \partial_{z} \psi\right\}$ is natural since $\gamma=Q / f_{1}$ and $\partial_{z} \psi=M / f_{2}$, where $Q$ is the shear force and $M$ is the bending moment. This makes boundary values easy to express.

It is convenient to write $(2.2)$ as

$$
\partial_{z} \boldsymbol{u}=\mathcal{D} \boldsymbol{u} .
$$

$\mathcal{D}$ is an integro-differential matrix operator, which may be subdivided into three parts $\mathcal{D}=$ $\mathcal{D}_{0}+\mathcal{D}_{1}+\mathcal{D}_{2}$. The first term, $\mathcal{D}_{0}$, represents the dynamics of a free (unrestrained), homogeneous beam. $\mathcal{D}_{1}$ models the influence of the restraining viscoelastic layer while the spatial dependence of the shear and bending stiffnesses is contained in $\mathcal{D}_{2}$.

\section{The wave splitting transformation}

The wave splitting transformation is performed in order to diagonalize the Timoshenko equation of a free, homogeneous beam [1]. At a fixed cross section, the wave fields of such a beam are decomposed into pairs of uncoupled right and left moving waves: $u_{1}^{+}, u_{2}^{+}$and $u_{1}^{-}, u_{2}^{-}$. Inside a region of inhomogeneity these waves couple and the interpretation of purely right and left moving fields is in general not valid. However, the wave splitting transformation remains a suitable mathematical tool for studying scattering by inhomogeneities.

The part of (2.3) that represents a free, homogeneous beam is $\partial_{z} \boldsymbol{u}=\mathcal{D}_{0} \boldsymbol{u}$. Introduce a wave splitting operator $\mathcal{B}$ and its formal inverse $\mathcal{B}^{-1}$ according to $\boldsymbol{w}=\mathcal{B} \boldsymbol{u}$ and $\boldsymbol{u}=\mathcal{B}^{-1} \boldsymbol{w}$, where $\boldsymbol{w}=\left(u_{1}^{+}, u_{2}^{+}, u_{1}^{-}, u_{2}^{-}\right)^{T}$. The matrix operator $\mathcal{B}$ is chosen so as to diagonalize $\mathcal{D}_{0}$ through 
$\mathcal{B D}_{0} \mathcal{B}^{-1}=\operatorname{diag}\left(-\lambda_{1},-\lambda_{2}, \lambda_{1}, \lambda_{2}\right)=\boldsymbol{\Lambda}$.

Since $\partial_{z} \mathcal{B}^{-1}=0$ for a homogeneous beam, the equations of motions are diagonalized

$$
\partial_{z} \boldsymbol{w}=\boldsymbol{\Lambda} \boldsymbol{w}
$$

The $\lambda_{i}$ :s are the eigenoperators of $\mathcal{D}_{0}$, which are represented by $\lambda_{i}=\left(1 / c_{i}\right) \partial_{t}+F_{i} *$. Expressions of the integro-differential operators $\mathcal{B}$ and $\mathcal{B}^{-1}$ and the kernels $F_{i}(t)$ can be found in [3]. Note that the $F_{i}(t)$ are of exponential order $1 / \tau$, where the characteristic time $\tau$ is defined by $\tau=$ $\left(1 / 2 c_{1}\right)\left(1-c_{1}^{2} / c_{2}^{2}\right) \sqrt{f_{2} / f_{1}}$, which causes the split fields to increase exponentially with time [2]. Explicitly, (3.1) reads

$$
\left(\partial_{z} \pm\left(1 / c_{i}\right) \partial_{t}\right) u_{i}^{ \pm} \pm F_{i} * u_{i}^{ \pm}=0, \quad i=1,2
$$

Hence, it is clear that the split fields satisfy uncoupled one-way wave equations. The right moving waves, $u_{i}^{+}$, propagate in the positive $z$-direction with the wave-front velocity $c_{i}$, while the left moving counterparts, $u_{i}^{-}$, propagate in the opposite direction. This transformation is also valid for a restrained, inhomogeneous beam but then the system of equations is no longer diagonal.

\subsection{Application to the inhomogeneous equation}

Application of the wave splitting transformation $\boldsymbol{w}=\boldsymbol{B} \boldsymbol{u}$ to (2.3) leads to

$$
\partial_{z} \boldsymbol{w}=(\boldsymbol{\Lambda}+\mathcal{L}) \boldsymbol{w}
$$

where

$$
\Lambda=\mathcal{B D}_{0} \mathcal{B}^{-1}, \quad \mathcal{L}=\mathcal{B D}_{1} \mathcal{B}^{-1}+\mathcal{B D}_{2} \mathcal{B}^{-1}-\mathcal{B}\left(\partial_{z} \mathcal{B}^{-1}\right)
$$

The operator $\mathcal{L}$ represents the coupling between the split fields in a restrained and/or inhomogeneous beam. By introducing the two-by-two submatrices $\mathbf{C}=\operatorname{diag}\left(c_{1}, c_{2}\right)$ and $\mathbf{F}=\operatorname{diag}\left(F_{1}, F_{2}\right)$, the eigenmatrix $\boldsymbol{\Lambda}$ may be written $\boldsymbol{\Lambda}=\operatorname{diag}\left(-\mathbf{C}^{-1} \partial_{t}+\mathbf{F} *, \mathbf{C}^{-1} \partial_{t}+\mathbf{F} *\right)$. Thus, (3.2) is explicitly 
decomposed into coupled equations for the right and left moving fields

$$
\begin{aligned}
& \left(\mathbf{I} \partial_{z}+\mathbf{C}^{-1} \partial_{t}\right) \boldsymbol{u}^{+}=\left(\mathbf{L}_{11}-\mathbf{F} *\right) \boldsymbol{u}^{+}+\mathbf{L}_{12} \boldsymbol{u}^{-} \\
& \left(\mathbf{I} \partial_{z}-\mathbf{C}^{-1} \partial_{t}\right) \boldsymbol{u}^{-}=\mathbf{L}_{21} \boldsymbol{u}^{+}+\left(\mathbf{L}_{22}+\mathbf{F} *\right) \boldsymbol{u}^{-}
\end{aligned}
$$

where $\mathbf{L}_{i j}$ are the submatrices of $\mathcal{L}$. The two-by-two identity matrix is denoted $\mathbf{I}$. These coupled equations are the Timoshenko equations expressed in terms of the transformed fields $\boldsymbol{u}^{+}(z, t)=$ $\left(u_{1}^{+}, u_{2}^{+}\right)^{T}$ and $\boldsymbol{u}^{-}(z, t)=\left(u_{1}^{-}, u_{2}^{-}\right)^{T}$. The matrix $\mathcal{L}$ may contain both convolutional operators and multiplicative functions. Therefore the following partition is introduced

$$
\mathbf{L}_{i j}=\mathbf{M}_{i j}(z, \cdot) *+\mathbf{N}_{i j}(z)
$$

where the elements are denoted $\left(\mathbf{M}_{i j}\right)_{k l}=M_{i j k l}$ and $\left(\mathbf{N}_{i j}\right)_{k l}=N_{i j k l}$.

\subsection{Travel time coordinates}

It is convenient to express the dynamics (3.4) on non-dimensional form. This is done by introducing the travel time coordinate transformation

$$
z^{\prime}(z)=\frac{1}{l} \int_{0}^{z} \frac{d z^{\prime \prime}}{c_{2}\left(z^{\prime \prime}\right)} \quad z^{\prime} \in[0,1], \quad s(t)=\frac{t}{l}, \quad l=\int_{0}^{d} \frac{d z^{\prime \prime}}{c_{2}\left(z^{\prime \prime}\right)}
$$

where $l$ is the time for the faster wave front to traverse the full length $d$ of the inhomogeneity. The non-dimensional velocities for the wave fronts are chosen such that $c_{2}{ }^{\prime}=1$ and $c_{1}{ }^{\prime}=c_{1} / c_{2}=\alpha$. Thus, the non-dimensional travel time for the slower wave front to traverse the distance between $x$ and $y, 0 \leq x, y \leq 1$, is

$$
\sigma(y, x)=\int_{x}^{y} \frac{1}{\alpha\left(z^{\prime \prime}\right)} d z^{\prime \prime}
$$

By transforming the operators to non-dimensional form and dropping the primes, the dynamics (3.4) are

$$
\begin{aligned}
& \left(\mathbf{I} \partial_{z}+\mathbf{C}^{-1} \partial_{s}\right) \boldsymbol{u}^{+}=\mathbf{N}_{11} \boldsymbol{u}^{+}+\mathbf{N}_{12} \boldsymbol{u}^{-}+\left(\mathbf{M}_{11}-\mathbf{F}\right) * \boldsymbol{u}^{+}+\mathbf{M}_{12} * \boldsymbol{u}^{-} \\
& \left(\mathbf{I} \partial_{z}-\mathbf{C}^{-1} \partial_{s}\right) \boldsymbol{u}^{-}=\mathbf{N}_{21} \boldsymbol{u}^{+}+\mathbf{N}_{22} \boldsymbol{u}^{-}+\mathbf{M}_{21} * \boldsymbol{u}^{+}+\left(\mathbf{M}_{22}+\mathbf{F}\right) * \boldsymbol{u}^{-}
\end{aligned}
$$




\section{The propagator representation}

As an incident field impinges on the region of inhomogeneity, it is scattered into coupled left and right moving fields. Consider waves that are generated in the homogeneous region $z<0$ and propagate in the positive $z$-direction towards the left boundary of the scattering region, $z=0$ (Figure 2). The scattered fields at an internal position $y(y>0)$ are related to the $\boldsymbol{u}^{+}$-field at $x(x>0)$ through linear operators known as wave propagators [10]. These are defined by the following mapping properties

$$
\boldsymbol{u}^{ \pm}(y, s+y-x)=\mathcal{P}^{ \pm+}(y, x) \boldsymbol{u}^{+}(x, s)
$$

The case in which all sources are present in the homogeneous region $z>1$ is studied in Appendix A. Note that there are no restrictions on the relative magnitudes of $x$ and $y$. When $y<x$, the fields are propagated backward in both time and space.

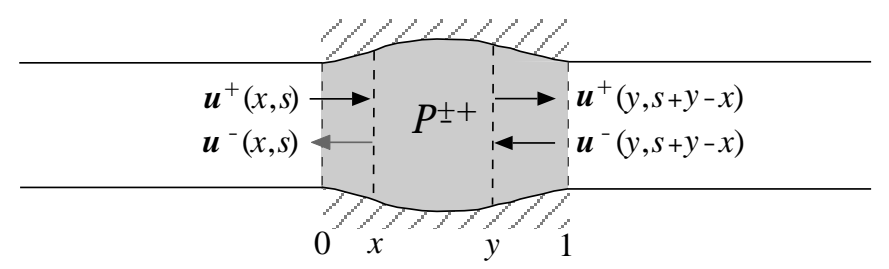

Figure 2: Scattering from sources in $z<0$.

The definition of the wave propagators implies the following relations for positive $x, x^{\prime}$ and $y$

$$
\begin{aligned}
& \mathcal{P}^{ \pm+}(y, x)=\mathcal{P}^{ \pm+}\left(y, x^{\prime}\right) \mathcal{P}^{++}\left(x^{\prime}, x\right) \\
& \mathcal{P}^{++}(x, x)=\mathcal{P}^{++}\left(x, x^{\prime}\right) \mathcal{P}^{++}\left(x^{\prime}, x\right)=\mathcal{I} .
\end{aligned}
$$

The last relation suggests the existence of an inverse operator according to $\left(\mathcal{P}^{++}(y, x)\right)^{-1}=$ $\mathcal{P}^{++}(x, y)$.

The functional form of the propagators is obtained by making use of the canonical representation [3]

$$
\boldsymbol{u}^{ \pm}(y, s)=\int_{-\infty}^{+\infty} \mathbf{U}^{ \pm}\left(x ; y, s-s^{\prime}\right) \boldsymbol{u}^{+}\left(x, s^{\prime}\right) d s^{\prime}
$$

where $\mathbf{U}^{ \pm}(x ; y, s)$ is the impulse response at $y$ originating from a right moving field at $x$ at time $s=0$. While all the responses may contain jump discontinuities, it is only the elements $U_{i i}^{+}$that 
carry Dirac's $\delta$-distributions along their respective characteristics

$$
\begin{aligned}
& U_{11}^{+}(x ; y, s)=t_{1}^{+}(y, x) \delta(s-\sigma(y, x))+U_{11}^{+}(x ; y, s)^{0} \\
& U_{22}^{+}(x ; y, s)=t_{2}^{+}(y, x) \delta(s-y+x)+U_{22}^{+}(x ; y, s)^{0} .
\end{aligned}
$$

The superscript 0 refers to the regular parts of the impulse responses $U_{i j}^{+}$. Inserting (4.5) into (4.4) and performing a shift in time, $s \rightarrow s+y-x$, gives

$$
\begin{aligned}
& \boldsymbol{u}^{+}(y, s+y-x)=\boldsymbol{t}^{+}(y, x) \mathcal{S}(y, x) \boldsymbol{u}^{+}(x, s)+\left(\mathbf{P}^{++}(y, x, \cdot) * \boldsymbol{u}^{+}(x, \cdot)\right)(s), \\
& \boldsymbol{u}^{-}(y, s+y-x)=\left(\mathbf{P}^{-+}(y, x, \cdot) * \boldsymbol{u}^{+}(x, \cdot)\right)(s),
\end{aligned}
$$

where the propagator kernels are defined as $\mathbf{P}^{ \pm+}(y, x, s)=\mathbf{U}^{ \pm}(x ; y, s+y-x)^{0}$. Note that the time variable is local in this definition; $s=0$ when waves corresponding to the faster velocity $c_{2}$ reach $y$ from $x$.

The elements in the diagonal matrix $\boldsymbol{t}^{+}(y, x)=\operatorname{diag}\left(t_{1}^{+}(y, x), t_{2}^{+}(y, x)\right)$ are the wave fronts factors that state the attenuation of the direct propagated part in the representation of the $\boldsymbol{u}^{+}-$ field in (4.6). Equations for these elements are obtained by studying the step function responses. This results in $t_{1}^{+}(y, x)=e^{\int_{x}^{y} N_{1111}\left(y^{\prime}\right) d y^{\prime}}$ and $t_{2}^{+}(y, x)=e^{\int_{x}^{y} N_{1122}\left(y^{\prime}\right) d y^{\prime}}$. Here the order of the arguments of the wave front factors is shifted as compared to that of [3]. The time shift matrix $\mathcal{S}(y, x)$ introduced in (4.6) is represented as $\mathcal{S}(y, x)=\operatorname{diag}\left(\mathcal{S}_{1}(y, x), \mathcal{S}_{0}(y, x)\right)$. The scalar valued time shift operators are defined $\mathcal{S}_{i}(y, x) f(x, s)=f\left(x, s-d_{i}^{+}(y, x)\right)$, where $d_{0}^{+}(y, x)$ and $d_{1}^{+}(y, x)$ measure the time elapsed after the arrival of the fast and the slow wave front at $y$, respectively. Hence, $d_{0}^{+}(y, x)=0$ and $d_{1}^{+}(y, x)=\sigma(y, x)-y+x$ where $\sigma(y, x)$ is defined in (3.6).

The existence of the inverse operator $\mathcal{P}^{++}(x, y, s)$ is guaranteed by the representation of the $\boldsymbol{u}^{+}$-field (4.6). Making use of (4.3) and the representation of the right moving field (4.6), leads to a set of Volterra equations of the second kind through which the propagator kernels $\mathbf{P}^{++}(x, y, s)$ and $\mathbf{P}^{++}(y, x, s)$ are related

$$
\begin{aligned}
\mathbf{P}^{++}(x, y, s) & +\boldsymbol{t}^{+}(x, y) \mathcal{S}(x, y)\left(\boldsymbol{t}^{+}(x, y) \mathcal{S}(x, y) \mathbf{P}^{++}(y, x, s)^{T}\right)^{T} \\
& +\boldsymbol{t}^{+}(x, y) \mathcal{S}(x, y)\left(\mathbf{P}^{++}(x, y, \cdot) * \mathbf{P}^{++}(y, x, \cdot)\right)(s)=0 .
\end{aligned}
$$




\section{$5 \quad$ Equations for the propagator kernels}

In this section the equations for the propagator kernels are derived. This is done by using the dynamics (3.7) and the representations (4.6). Note that different time arguments are used in these equations, the space and time derivatives being related as

$\left.\partial_{s} \boldsymbol{u}^{ \pm}(y, s)\right|_{s=s+y-x}=\partial_{s} \boldsymbol{u}^{ \pm}(y, s+y-x),\left.\quad \partial_{y} \boldsymbol{u}^{ \pm}(y, s)\right|_{s=s+y-x}=\left(\partial_{y}-\partial_{s}\right) \boldsymbol{u}^{ \pm}(y, s+y-x)$.

Partial differentiation of the $\boldsymbol{u}^{+}$-representation (4.6) with respect to $s$ and $y$ gives

$$
\begin{aligned}
& \partial_{s} \boldsymbol{u}^{+}(y, s+y-x)=\boldsymbol{t}^{+} \partial_{s}\left(\mathcal{S} \boldsymbol{u}^{+}\right)+\partial_{s}\left(\mathbf{P}^{++} * \boldsymbol{u}^{+}\right), \\
& \partial_{y} \boldsymbol{u}^{+}(y, s+y-x)=\partial_{y} \boldsymbol{t}^{+} \boldsymbol{S} \boldsymbol{u}^{+}+\boldsymbol{t}^{+}\left(\mathbf{I}-\mathbf{C}^{-1}\right) \partial_{s}\left(\mathcal{S} \boldsymbol{u}^{+}\right)+\partial_{y}\left(\mathbf{P}^{++} * \boldsymbol{u}^{+}\right) .
\end{aligned}
$$

while the corresponding partial derivatives of the $\boldsymbol{u}^{-}$-representation (4.6) is straightforward. If nothing else is stated $\alpha, \boldsymbol{N}, \boldsymbol{t}^{+}, \mathcal{S}, \boldsymbol{u}^{+}, \boldsymbol{M}$ and $\mathbf{P}^{ \pm+}$are implicitly taken to be $\alpha(y), \boldsymbol{N}(y)$, $\boldsymbol{t}^{+}(y, x), \mathcal{S}(y, x), \boldsymbol{u}^{+}(x, s), \boldsymbol{M}(y, s)$ and $\mathbf{P}^{ \pm+}(y, x, s)$, respectively.

The elements of the propagator kernels may contain jump discontinuities across a set of curves $s=d_{i}^{+}(y, x)$. These curves are members of the families of characteristic curves of the propagator kernels and the corresponding discontinuities are denoted

$$
\left[\mathbf{P}^{ \pm+}\right]_{i}=\mathbf{P}^{ \pm+}\left(y, x, d_{i}^{+}(y, x)^{+}\right)-\mathbf{P}^{ \pm+}\left(y, x, d_{i}^{+}(y, x)^{-}\right)
$$

The time and space derivatives of the temporal convolutions above are thus

$$
\begin{aligned}
& \partial_{s}\left(\mathbf{P}^{ \pm+} * \boldsymbol{u}^{+}\right)=\partial_{s} \mathbf{P}^{ \pm+} * \boldsymbol{u}^{+}+\sum_{i=0}^{n}\left[\mathbf{P}^{ \pm+}\right]_{i} \boldsymbol{u}^{+}\left(x, s-d_{i}^{+}\right), \\
& \partial_{y}\left(\mathbf{P}^{ \pm+} * \boldsymbol{u}^{+}\right)=\partial_{y} \mathbf{P}^{ \pm+} * \boldsymbol{u}^{+}-\sum_{i=0}^{n}\left(\partial_{y} d_{i}^{+}\right)\left[\mathbf{P}^{ \pm+}\right]_{i} \boldsymbol{u}^{+}\left(x, s-d_{i}^{+}\right),
\end{aligned}
$$

where the distributional parts of the integrands have been extracted on the right-hand side. Inserting the representations (4.6) into the dynamics (3.7) and then making use of the relations (5.1) results in equations consisting of convolutions with $\boldsymbol{u}^{+}$and sums over contributions from the curves of discontinuity. These parts can be proven independent and therefore must be treated 
separately [3]. The propagator equations originate from the convolutional parts and read

$$
\begin{aligned}
& \partial_{y} \mathbf{P}^{++}+\left(\mathbf{C}^{-1}-\mathbf{I}\right) \partial_{s} \mathbf{P}^{++}=\left(\mathcal{S}\left(\mathbf{M}_{11}^{T}-\mathbf{F}\right)\right)^{T} \boldsymbol{t}^{+} \\
& +\mathbf{N}_{11} \mathbf{P}^{++}+\mathbf{N}_{12} \mathbf{P}^{-+}+\left(\mathbf{M}_{11}-\mathbf{F}\right) * \mathbf{P}^{++}+\mathbf{M}_{12} * \mathbf{P}^{-+} \\
& \partial_{y} \mathbf{P}^{-+}-\left(\mathbf{C}^{-1}+\mathbf{I}\right) \partial_{s} \mathbf{P}^{-+}=\left(\mathcal{S} \mathbf{M}_{21}^{T}\right)^{T} \boldsymbol{t}^{+} \\
& +\mathbf{N}_{21} \mathbf{P}^{++}+\mathbf{N}_{22} \mathbf{P}^{-+}+\mathbf{M}_{21} * \mathbf{P}^{++}+\left(\mathbf{M}_{22}+\mathbf{F}\right) * \mathbf{P}^{-+}
\end{aligned}
$$

The equations for the discontinuities are

$$
\begin{aligned}
& \mathbf{N}_{11}^{0} \boldsymbol{t}^{+} \mathcal{S} \boldsymbol{u}^{+}-\sum_{i=0}^{n}\left(\mathbf{C}^{-1}-\mathbf{I}-\left(\partial_{y} d_{i}^{+}\right) \mathbf{I}\right)\left[\mathbf{P}^{++}\right]_{i} \boldsymbol{u}^{+}\left(x, s-d_{i}^{+}\right)=0 \\
& \mathbf{N}_{21} \boldsymbol{t}^{+} \mathcal{S} \boldsymbol{u}^{+}+\sum_{i=0}^{n}\left(\mathbf{C}^{-1}+\mathbf{I}+\left(\partial_{y} d_{i}^{+}\right) \mathbf{I}\right)\left[\mathbf{P}^{-+}\right]_{i} \boldsymbol{u}^{+}\left(x, s-d_{i}^{+}\right)=0
\end{aligned}
$$

where $\mathbf{N}_{11}^{0}=\mathbf{N}_{11}-\operatorname{diag}\left(N_{1111}, N_{1122}\right)$.

The propagator kernels are independent of the incoming and the scattered fields and depend solely on the properties of the region of inhomogeneity. Note that the relations above are valid without any restrictions on the relative magnitudes of $x$ and $y$. Moreover, in order for the solutions of (5.2) and (5.3) to be causal and the representations (4.6) to be consistent with the scattering problem, the following boundary and initial values must hold for the propagator kernels

$$
\begin{aligned}
& \mathbf{P}^{++}(x, x, s)=\mathbf{P}^{-+}(1, x, s)=0, \quad \forall s>0 \quad(\text { from (4.6)) }, \\
& \mathbf{P}^{++}\left(y, x, 0^{-}\right)=\mathbf{P}^{-+}\left(y, x, 0^{-}\right)=0, \quad y>x \quad \text { (by causality). }
\end{aligned}
$$

\section{Discontinuities of the propagator kernels}

From the definition of the time shift matrix, it is clear that the propagator kernels may be discontinuous across the characteristics $d_{0}^{+}$and $d_{1}^{+}$emanating from $(z, s)=(x, 0)$. These curves are marked by solid lines in Figure 3 .

The magnitude of the jump discontinuities of $\mathbf{P}^{++}$that are obtained directly from (5.4) are

$$
\left[P_{12}^{++}\right]_{0}=\frac{\alpha}{1-\alpha} N_{1112} t_{2}^{+}, \quad\left[P_{21}^{++}\right]_{1}=\frac{-\alpha}{1-\alpha} N_{1121} t_{1}^{+},
$$




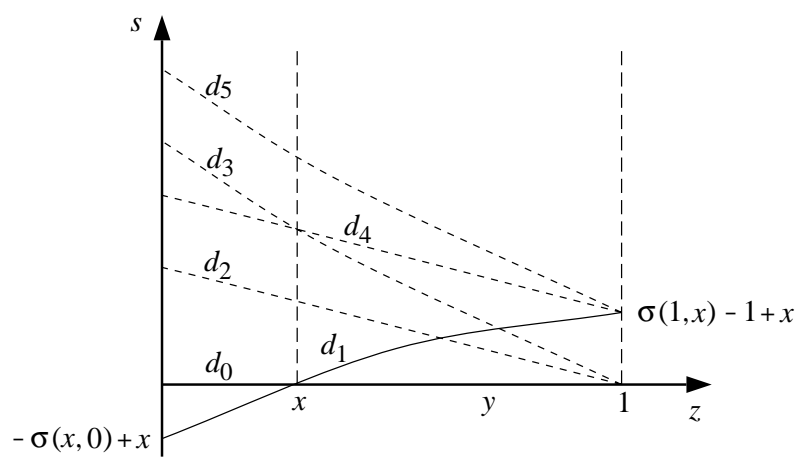

Figure 3: The discontinuity characteristics of $\mathbf{P}^{++}$.

and $\left[P_{11}^{++}\right]_{0}=\left[P_{22}^{++}\right]_{1}=0$. The remaining possible discontinuities in $\mathbf{P}^{++}$across its characteristics $d_{0}^{+}$and $d_{1}^{+}$are yet to be determined. As for $\mathbf{P}^{-+}$, all the possible jumps across $d_{0}^{+}$and $d_{1}^{+}$ are obtained directly from (5.5). The non-zero ones are

$$
\begin{array}{ll}
{\left[P_{11}^{-+}\right]_{1}=\frac{-\alpha}{2} N_{2111} t_{1}^{+},} & {\left[P_{12}^{-+}\right]_{0}=\frac{-\alpha}{1+\alpha} N_{2112} t_{2}^{+},} \\
{\left[P_{21}^{-+}\right]_{1}=\frac{-\alpha}{1+\alpha} N_{2121} t_{1}^{+},} & {\left[P_{22}^{-+}\right]_{0}=\frac{-1}{2} N_{2122} t_{2}^{+},}
\end{array}
$$

while $\left[P_{11}^{-+}\right]_{0}=\left[P_{21}^{-+}\right]_{0}=\left[P_{12}^{-+}\right]_{1}=\left[P_{22}^{-+}\right]_{1}=0$. The undetermined jump discontinuities of $\mathbf{P}^{++}$can be derived from (5.2) together with the consistency requirements on the jumps (5.6) as $y$ approaches $x$ from the right. In this limit, the sum of all jumps of the respective kernels must equal zero

$$
\left.\left[P_{i j}^{++}\right]_{0}\right|_{y \rightarrow x}+\left.\left[P_{i j}^{++}\right]_{1}\right|_{y \rightarrow x}=0,
$$

which, using (6.1), gives

$$
\left.\left[P_{12}^{++}\right]_{1}\right|_{y \rightarrow x}=\frac{-\alpha(x)}{1-\alpha(x)} N_{1112}(x),\left.\quad\left[P_{21}^{++}\right]_{0}\right|_{y \rightarrow x}=\frac{\alpha(x)}{1-\alpha(x)} N_{1121}(x),
$$

together with $\left.\left[P_{11}^{++}\right]_{1}\right|_{y \rightarrow x}=\left.\left[P_{22}^{++}\right]_{0}\right|_{y \rightarrow x}=0$. The transport equations for the jump discontinuities are obtained from (5.2) making use of the fact that the left-hand side derivatives are the 
directional derivatives along the characteristics corresponding to $d_{0}^{+}$and $d_{1}^{+}$. Consequently,

$$
\begin{array}{lll}
\frac{d}{d y}\left[P_{11}^{++}\right]_{1}=N_{1111}\left[P_{11}^{++}\right]_{1}+f_{11}^{+} t_{1}^{+}, & \frac{d}{d y}\left[P_{12}^{++}\right]_{1}=N_{1111}\left[P_{12}^{++}\right]_{1}, \\
\frac{d}{d y}\left[P_{21}^{++}\right]_{0}=N_{1122}\left[P_{21}^{++}\right]_{0}, & \frac{d}{d y}\left[P_{22}^{++}\right]_{0}=N_{1122}\left[P_{22}^{++}\right]_{0}+f_{22}^{+} t_{2}^{+},
\end{array}
$$

where

$$
\begin{aligned}
& f_{11}^{+}(y)=M_{1111}\left(y, 0^{+}\right)-F_{1}\left(y, 0^{+}\right)-\frac{\alpha}{2} N_{1211} N_{2111}-\frac{\alpha}{1-\alpha} N_{1112} N_{1121}-\frac{\alpha}{1+\alpha} N_{1212} N_{2121} \\
& f_{22}^{+}(y)=M_{1122}\left(y, 0^{+}\right)-F_{2}\left(y, 0^{+}\right)-\frac{1}{2} N_{1222} N_{2122}+\frac{\alpha}{1-\alpha} N_{1112} N_{1121}-\frac{\alpha}{1+\alpha} N_{1221} N_{2112} .
\end{aligned}
$$

By integrating (6.5), together with the boundary values stated in (6.4), the remaining jumps of $\mathbf{P}^{++}$are

$$
\begin{array}{ll}
{\left[P_{11}^{++}\right]_{1}=t_{1}^{+} \int_{x}^{y} f_{11}^{+}\left(y^{\prime}\right) d y^{\prime},} & {\left[P_{12}^{++}\right]_{1}=\frac{-\alpha(x)}{1-\alpha(x)} N_{1112}(x) t_{1}^{+},} \\
{\left[P_{21}^{++}\right]_{0}=\frac{\alpha(x)}{1-\alpha(x)} N_{1121}(x) t_{2}^{+},} & {\left[P_{22}^{++}\right]_{0}=t_{2}^{+} \int_{x}^{y} f_{22}^{+}\left(y^{\prime}\right) d y^{\prime} .}
\end{array}
$$

This far, all the possible discontinuities of $\mathbf{P}^{++}$are given above, as well as some of the possible discontinuities of $\mathbf{P}^{-+}$. However, it is clear from (5.5) that $\mathbf{P}^{-+}$may have jumps across curves with slopes $\partial_{y} d^{+}=-2$ and $\partial_{y} d^{+}=-(1+\alpha) / \alpha$. These describe the families of characteristic curves of (5.3). The discontinuity characteristics that may emanate from the intersections of $d_{0}^{+}$ and $d_{1}^{+}$with $y=1$ are ( Figure 4 )

$$
\begin{array}{ll}
d_{2}^{+}(y, x)=2-2 y, & d_{3}^{+}(y, x)=\sigma(1, y)+1-y, \\
d_{4}^{+}(y, x)=\sigma(1, x)+1-2 y+x, & d_{5}^{+}(y, x)=\sigma(1, y)+\sigma(1, x)-y+x .
\end{array}
$$

From (5.5) it follows that

$$
\left[P_{1 j}^{-+}\right]_{2}=\left[P_{1 j}^{-+}\right]_{4}=\left[P_{2 j}^{-+}\right]_{3}=\left[P_{2 j}^{-+}\right]_{5}=0, \quad j=1,2
$$

The remaining jump discontinuities of $\mathbf{P}^{-+}$are determined by using the corresponding propagator 


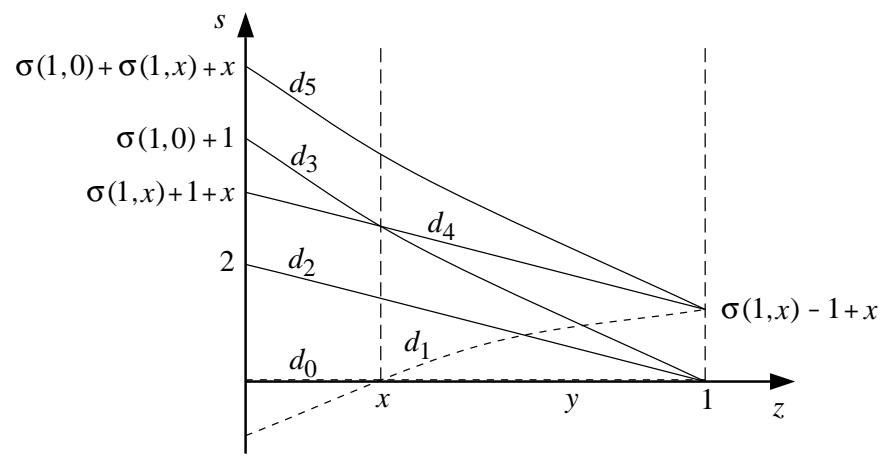

Figure 4: The additional discontinuity characteristics of $\mathbf{P}^{-+}$.

equation (5.3) together with the consistency requirements on the jumps in the limit $y \rightarrow 1$

$$
\begin{aligned}
& {\left.\left[P_{i j}^{-+}\right]_{0}\right|_{y \rightarrow 1}+\left.\left[P_{i j}^{-+}\right]_{2}\right|_{y \rightarrow 1}+\left.\left[P_{i j}^{-+}\right]_{3}\right|_{y \rightarrow 1}=0,} \\
& {\left.\left[P_{i j}^{-+}\right]_{1}\right|_{y \rightarrow 1}+\left.\left[P_{i j}^{-+}\right]_{4}\right|_{y \rightarrow 1}+\left.\left[P_{i j}^{-+}\right]_{5}\right|_{y \rightarrow 1}=0 .}
\end{aligned}
$$

These are the analogies of (6.3). The jump discontinuities not given in (6.6) are obtained in the same manner as for the jumps of $\mathbf{P}^{++}$. Hence

$$
\begin{array}{ll}
{\left[P_{11}^{-+}\right]_{5}=\frac{\alpha(1)}{2} N_{2111}(1) t_{1}^{+}(1, x) t_{1}^{-}(y, 1),} & {\left[P_{12}^{-+}\right]_{3}=\frac{\alpha(1)}{1+\alpha(1)} N_{2112}(1) t_{2}^{+}(1, x) t_{1}^{-}(y, 1),} \\
{\left[P_{21}^{-+}\right]_{4}=\frac{\alpha(1)}{1+\alpha(1)} N_{2121}(1) t_{1}^{+}(1, x) t_{2}^{-}(y, 1),} & {\left[P_{22}^{-+}\right]_{2}=\frac{1}{2} N_{2122}(1) t_{2}^{+}(1, x) t_{2}^{-}(y, 1),}
\end{array}
$$

and $\left[P_{11}^{-+}\right]_{3}=\left[P_{12}^{-+}\right]_{5}=\left[P_{21}^{-+}\right]_{2}=\left[P_{22}^{-+}\right]_{4}=0$. The functions $t_{i}^{-}(y, x)$ are the elements of the wave front matrix $\boldsymbol{t}^{-}(y, x)$ defined in Appendix A.

\section{Related techniques}

The wave propagators are related to other time domain formalisms such as the Green function technique and the imbedding method. This section presents these approaches as special cases of the propagator technique. 


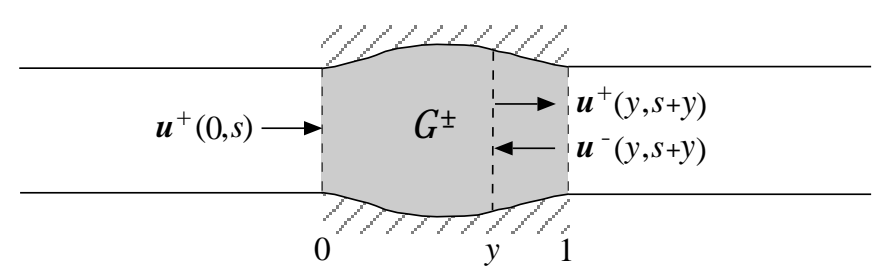

Figure 5: The Green function approach.

\subsection{The Green function technique}

In the Green function approach operators $\mathcal{G}^{ \pm}$map the incident fields at $z=0$ to the scattered ones at $y>0$ (Figure 5 ) as

$$
\boldsymbol{u}^{ \pm}(y, s+y)=\mathcal{G}^{ \pm}(y) \boldsymbol{u}^{+}(0, s)
$$

By comparing this to the definition of the propagators (4.1), it is clear that $\mathcal{G}^{ \pm}(y)=\mathcal{P}^{ \pm+}(y, 0)$, and the kernels are related through $\mathbf{G}^{ \pm}(y, s)=\mathbf{P}^{ \pm+}(y, 0, s)$, implying that the equations and the jump conditions for $\mathbf{G}^{ \pm}(y, s)$ follow from Section 5 and Section 6 with $x=0$. In [2], Green functions are obtained for a free homogeneous beam.

\subsection{The imbedding method}

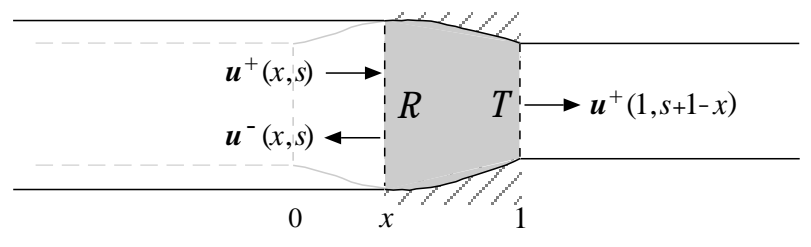

Figure 6: The imbedding geometry.

The imbedding approach covers reflection and transmission on a subregion $z \in[x, 1]$ of the whole region of inhomogeneity $z \in[0,1]$ (Figure 6). Introducing a fictitious homogeneous continuation to the left of $z=x$, the scattering problem of the full region is imbedded in a one-parameter family of scattering problems. The operators $\mathcal{R}$ and $\mathcal{T}$ are defined by

$$
\boldsymbol{u}^{-}(x, s)=\mathcal{R}(x) \boldsymbol{u}^{+}(x, s), \quad \boldsymbol{u}^{+}(1, s+1-x)=\mathcal{T}(x) \boldsymbol{u}^{+}(x, s)
$$

Apparently, $\mathcal{R}(x)=\mathcal{P}^{-+}(x, x)$ and $\mathcal{T}(x)=\mathcal{P}^{++}(1, x)$. Moreover, the kernel relations become $\mathbf{R}(x, s)=\mathbf{P}^{-+}(x, x, s)$ and $\mathbf{T}(x, s)=\mathbf{P}^{++}(1, x, s)$. The equations for $\mathbf{R}(x, s)$ and $\mathbf{T}(x, s)$ are 
obtained from propagator equations where the spatial derivatives are taken with respect to the second variable $x$ instead of $y$ as in Section 5. In [3] the imbedding equations are derived with the transmission kernel $\mathbf{T}$ being defined in a slightly different manner than above.

\section{Symmetry properties illustrated by two examples}

This section deals with the various symmetries that exists for the coupling functions (3.5). Their explicit representations, together with the discontinuities of the propagator kernels, are given for two different cases.

First consider the operator identity $\mathcal{L}=\mathcal{I} \partial_{z}-\boldsymbol{\Lambda}$ from (3.2), where $\mathcal{I}$ is a four-by-four identity matrix. The matrices $\mathbf{C}$ and $\mathbf{F}$ are independent under a reflection of the coordinate system in the

origin $\widetilde{z}=-z$. Thus, $\widetilde{\mathbf{F}}=\mathbf{F}, \widetilde{\mathbf{C}}=\mathbf{C}$ and thereby $\widetilde{\boldsymbol{\Lambda}}=\boldsymbol{\Lambda}$. Applying the change of frames gives $\widetilde{\mathcal{L}}=\mathcal{I} \partial_{\tilde{z}}-\widetilde{\boldsymbol{\Lambda}}=-\left(\mathcal{I} \partial_{z}+\boldsymbol{\Lambda}\right)$. By defining the matrix $\mathcal{A}$ according to

$$
\mathcal{A}=\left(\begin{array}{ll}
\text { 0 } & \text { I } \\
\text { I } & 0
\end{array}\right)
$$

and using $\mathcal{A}\left(\mathcal{I} \partial_{z}+\boldsymbol{\Lambda}\right)=\left(\mathcal{I} \partial_{z}-\boldsymbol{\Lambda}\right) \mathcal{A}$, the following general relation is obtained

$$
\widetilde{\mathcal{L}}=-\mathcal{A} \mathcal{L} \mathcal{A}
$$

Second, consider the operators constituting $\mathcal{L}$ as defined in (3.3). It is apparent that

$$
\widetilde{\mathcal{B}}=\mathcal{B}, \quad \widetilde{\mathcal{B}}^{-1}=\mathcal{B}^{-1}, \quad \widetilde{\mathcal{D}}_{1}=\mathcal{D}_{1},
$$

and, due to the presence of spatial derivatives,

$$
\widetilde{\mathcal{D}}_{2}=-\mathcal{D}_{2}, \quad \partial_{\tilde{z}} \widetilde{\mathcal{B}}^{-1}=-\partial_{z} \mathcal{B}^{-1}
$$

For a homogeneous beam that is suspended on a viscoelastic layer, it holds that $\mathcal{D}_{2}=\partial_{z} \mathcal{B}^{-1}=$ 0. Then from the definition of $\mathcal{L}$ and using (8.2), $\widetilde{\mathcal{L}}=\mathcal{L}$ and therefore $\widetilde{\mathbf{M}}_{i j}=\mathbf{M}_{i j}$ and $\widetilde{\mathbf{N}}_{i j}=\mathbf{N}_{i j}$. Combining this result with the general relation (8.1) gives the following symmetry requirements 
for the suspended, homogeneous beam

$$
\mathbf{M}_{11}=-\mathbf{M}_{22}, \quad \mathbf{M}_{12}=-\mathbf{M}_{21}, \quad \mathbf{N}_{11}=-\mathbf{N}_{22}, \quad \mathbf{N}_{12}=-\mathbf{N}_{21} .
$$

In the case of a free inhomogeneous beam, $\mathcal{D}_{1}=0$. Making use of $(8.3)$, it follows that $\widetilde{\mathcal{L}}=-\mathcal{L}$ which results in $\widetilde{\mathbf{M}}_{i j}=-\mathbf{M}_{i j}$ and $\widetilde{\mathbf{N}}_{i j}=-\mathbf{N}_{i j}$. Using (8.1), the symmetry requirements for the unrestrained, inhomogeneous beam are obtained as

$$
\mathbf{M}_{11}=\mathbf{M}_{22}, \quad \mathbf{M}_{12}=\mathbf{M}_{21}, \quad \mathbf{N}_{11}=\mathbf{N}_{22}, \quad \mathbf{N}_{12}=\mathbf{N}_{21} .
$$

\subsection{Viscoelastic damping of a homogeneous beam}

For a homogeneous beam that is suspended on a viscoelastic layer of length $d$, the material parameters are spatially independent and the operator $\mathcal{L}$ in (3.3) is reduced to $\mathcal{L}=\mathcal{B D}_{1} \mathcal{B}^{-1}$. The operator $\mathcal{L}$ is calculated by means of Laplace transform techniques as described in [2]. The results reveal that none of its matrix elements contain purely multiplicative terms, so the partition introduced in (3.5) simplifies to $\mathbf{L}_{i j}=\mathbf{M}_{i j}(\cdot) *$. The convolution kernels can be structured as

$$
\mathbf{M}_{11}=-\mathbf{M}_{22}=\chi_{1} \mathbf{A}_{1}+\chi_{2} \mathbf{A}_{2}, \quad \mathbf{M}_{12}=-\mathbf{M}_{21}=\chi_{1} \mathbf{A}_{1}-\chi_{2} \mathbf{A}_{2},
$$

where the operators $\chi_{i}$ are defined in Section 2 and the operator matrices $\mathbf{A}_{i}$ are found in [3]. Note that these coupling matrices possess the symmetries predicted in (8.4). Further, since $\mathbf{N}_{i j}=0$, the wave front matrices equal the identity matrix $\boldsymbol{t}^{+}=\boldsymbol{t}^{-}=\mathbf{I}$. According to Section 6 , the only non-zero jump discontinuities are given by

$$
\left[P_{11}^{++}\right]_{1}=\frac{d^{2}}{2}\left(-\alpha \frac{k_{1}}{f_{1}}+\frac{\alpha}{1-\alpha^{2}} \frac{f_{1}}{f_{2}}\right)(y-x), \quad\left[P_{22}^{++}\right]_{0}=-\frac{d^{2}}{2}\left(\frac{k_{2}}{f_{2}}+\frac{1}{1-\alpha^{2}} \frac{f_{1}}{f_{2}}\right)(y-x) .
$$

\subsection{Unsupported beam with a varying cross section}

The second example concerns an unsupported beam that has a region of length $d$ along which the dimensions of the cross section vary. The cross section is assumed to be either circular or rectangular, since these are examples of geometries where $k^{\prime}$ is independent of the cross sectional dimensions, [20]. Thus, the non-dimensional velocity $\alpha$ is constant along the beam. Since the beam is unsupported, $\mathcal{D}_{1}=0$ and the operator $\mathcal{L}=\mathcal{B D}_{2} \mathcal{B}^{-1}-\mathcal{B}\left(\partial_{z} \mathcal{B}^{-1}\right)$. The purely multiplicative 
matrices $\mathbf{N}_{i j}$ are given by

$$
\mathbf{N}_{11}(y)=\frac{1}{2}\left(\begin{array}{cc}
-\frac{A^{\prime}(y)}{A(y)} & -\frac{1}{\alpha} \frac{A^{\prime}(y)}{A(y)}+\frac{I^{\prime}(y)}{I(y)} \\
0 & -\frac{I^{\prime}(y)}{I(y)}
\end{array}\right), \quad \mathbf{N}_{12}(y)=\frac{1}{2}\left(\begin{array}{cc}
\frac{A^{\prime}(y)}{A(y)} & \frac{1}{\alpha} \frac{A^{\prime}(y)}{A(y)}+\frac{I^{\prime}(y)}{I(y)} \\
0 & -\frac{I^{\prime}(y)}{I(y)}
\end{array}\right),
$$

where $\mathbf{N}_{11}=\mathbf{N}_{22}$ and $\mathbf{N}_{12}=\mathbf{N}_{21}$. The matrices $\mathbf{M}_{i j}(y, s)$ may be expressed according to

$$
\mathbf{M}_{1 j}(y, s)=\frac{1}{2} \mu(y)\left(\frac{A^{\prime}(y)}{A(y)} \mathbf{A}_{\mathrm{M}, j}(\mu(y) s)+\frac{I^{\prime}(y)}{I(y)} \mathbf{B}_{\mathrm{M}, j}(\mu(y) s)\right)
$$

with $\mathbf{M}_{11}=\mathbf{M}_{22}$ and $\mathbf{M}_{12}=\mathbf{M}_{21}$. The function $\mu$ is defined by

$$
\mu(y)=\frac{l}{\tau}=\frac{2 \alpha^{2}}{1-\alpha^{2}} d \sqrt{\frac{A(y)}{I(y)}} .
$$

The expressions for the matrix-valued functions $\mathbf{A}_{\mathrm{M}, j}$ and $\mathbf{B}_{\mathrm{M}, j}$ are quite complicated and lengthy, involving modified Bessel functions [4]. Note that the coupling matrices $\mathbf{M}_{i j}$ and $\mathbf{N}_{i j}$ possess the symmetries predicted in (8.5).

With the elements $\mathbf{N}_{i j}$ as stated in (8.7), the wave front matrices are

$$
\boldsymbol{t}^{+}(y, x)=\boldsymbol{t}^{-}(y, x)=\operatorname{diag}\left(\sqrt{\frac{A(x)}{A(y)}}, \sqrt{\frac{I(x)}{I(y)}}\right)
$$

Since the multiplicative terms $\mathbf{N}_{i j}$ are non-zero, there are several jump discontinuities according to Section 6. These explicit expressions are straightforward to obtain, and thus not presented here.

\section{$9 \quad$ Numerical method}

This section describes an algorithm for solution of the partial integro-differential equations satisfied by the propagator kernels. A numerical example is presented in Section 10 for a homogeneous beam on a viscoelastic layer.

It has been found that the time dependent coupling matrices are of exponential order $1 / \tau$ [2], where $\tau$ is a characteristic time parameter of the beam and is defined in Section 3. In order to suppress numerical instabilities that occur in the numerical treatment of the equations for the propagator kernels, this exponential factor is extracted. Thus, introduce the transformation $\mathbf{P}^{ \pm+{ }^{\prime}}(y, x, s)=e^{-\mu(y) s} \mathbf{P}^{ \pm+}(y, x, s)$ together with $\mathbf{F}^{\prime}(y, s)=e^{-\mu(y) s} \mathbf{F}(y, s)$ and $\mathbf{M}_{i j}{ }^{\prime}(y, s)=$ 
$e^{-\mu(y) s} \mathbf{M}_{i j}(y, s)$. The argument of the exponential, $\mu(y) s=t / \tau(z)$, originates from the introduction of the non-dimensional variables in Section $3.2 ; \mu$ is the non-dimensional counterpart of $1 / \tau$. The time independent coupling matrices $\mathbf{N}_{i j}(y)$ remain unchanged, as do the wave front matrices $\boldsymbol{t}^{+}(y, x)$ and the curves of discontinuity $d_{i}^{+}(y, x)$. However, the time-shift operator is modified and now reads $\mathcal{S}^{\prime}(y, x)=\operatorname{diag}\left(e^{-\mu(y) d_{1}^{+}(y, x)} \mathcal{S}_{1}(y, x),, e^{-\mu(y) d_{0}^{+}(y, x)} \mathcal{S}_{0}(y, x)\right)$. Finally, the initial conditions and the boundary values (5.6) remain the same while the magnitudes of the jump discontinuities alter according to $\left[P_{i j}^{ \pm+^{\prime}}\right]_{k}=e^{-\mu(y) d_{k}^{+}(y, x)}\left[P_{i j}^{ \pm+}\right]_{k}$.

Dropping the primes, the equations (5.2) and (5.3) for the propagator kernels turn into

$$
\left(\mathbf{I} \partial_{y}+\left(\mathbf{C}^{-1}-\mathbf{I}\right) \partial_{s}\right) \mathbf{P}^{++}=\mathcal{J}^{+} \quad\left(\mathbf{I} \partial_{y}-\left(\mathbf{C}^{-1}+\mathbf{I}\right) \partial_{s}\right) \mathbf{P}^{-+}=\mathcal{J}^{-}
$$

where the right-hand sides are the following matrix-valued functionals

$$
\begin{aligned}
\mathcal{J}^{+} & =\left(\mathcal{S}\left(\mathbf{M}_{11}^{T}-\mathbf{F}\right)\right)^{T} \boldsymbol{t}^{+}+\left(\mathbf{N}_{11}-\mu^{\prime}(y) s \mathbf{I}+\mu(y)\left(\mathbf{I}-\mathbf{C}^{-1}\right)\right) \mathbf{P}^{++} \\
& +\mathbf{N}_{12} \mathbf{P}^{-+}+\left(\mathbf{M}_{11}-\mathbf{F}\right) * \mathbf{P}^{++}+\mathbf{M}_{12} * \mathbf{P}^{-+} \\
\mathcal{J}^{-} & =\left(\mathcal{S} \mathbf{M}_{21}^{T}\right)^{T} \boldsymbol{t}^{+}+\left(\mathbf{N}_{22}-\mu^{\prime}(y) s \mathbf{I}+\mu(y)\left(\mathbf{I}+\mathbf{C}^{-1}\right)\right) \mathbf{P}^{-+} \\
& +\mathbf{N}_{21} \mathbf{P}^{++}+\mathbf{M}_{21} * \mathbf{P}^{++}+\left(\mathbf{M}_{22}+\mathbf{F}\right) * \mathbf{P}^{-+}
\end{aligned}
$$

\subsection{Numerical integration of the propagator equations}

In the examples considered in Sections 8.1 and 8.2 it is assumed that the velocity ratio $\alpha$ is constant. Thus, $\sigma(y, x)=(y-x) / \alpha$ from (3.6). To form the mesh of calculation, the spatial interval $y \in[x, 1]$ is divided into $N$ subintervals. The time step, $h$, is subsequently chosen so that the characteristic curves corresponding to $d_{1}(x)$ coincide with a node point in the mesh at every time step, this means

$$
s_{k}=k h \quad k=0,1,2, \ldots, \quad y_{n}=x+n h_{y} \quad n=0,1,2, \ldots, N .
$$

The time step is related to the spatial step through $h=(1 / \alpha-1) h_{y}$ and $h_{y}=(1-x) / N$. The discretized kernel elements of $(9.1)$ are denoted $P_{i j, n k}^{ \pm+}=P_{i j}^{ \pm+}\left(y_{n}, x, s_{k}\right)$ and $P_{i j}^{ \pm+}(y)_{k}=$ $P_{i j}^{ \pm+}\left(y, x, s_{k}\right)$. The equation for each element is approximated along its family of characteristic curves, by using the method of Euler and the trapezoidal quadrature rule. These discretizations are used to set up a predictor-corrector procedure, in which Euler's method, the predictor, supplies 
a starting value for an iteration sequence which is generated by the trapezoidal rule, the corrector.

In the algorithm used here, a uniform calculation mesh is formed and linear interpolation is used to calculate data at points which are not on this mesh. Since the propagator kernels have jump discontinuities across the characteristic curves $d_{i}^{+}$, no interpolation is used across these curves. The values predicted by the method of Euler are, from (9.1),

$$
\begin{aligned}
& \left(P_{i j, n k}^{++}\right)^{(0)}=P_{i j}^{++}\left(y_{n-1}, x, s_{k-2+i}\right)+\frac{h}{\alpha_{i}^{+}} \mathcal{J}_{i j}^{+}\left(y_{n-1}, s_{k-2+i}\right), \\
& \left(P_{i j, n k}^{-+}\right)^{(0)}=P_{i j}^{-+}\left(Y_{i}, x, s_{k-1}\right)+\frac{h}{\alpha_{i}^{-}} \mathcal{J}_{i j}^{-}\left(Y_{i}, s_{k-1}\right) .
\end{aligned}
$$

Here, the factors $\alpha_{i}^{ \pm}$are the slopes of the characteristics of $P_{i j}^{++}$and $P_{i j}^{-+}$; that is $\alpha_{1}^{+}=1 / \alpha-$ $1, \alpha_{2}^{+}=0, \alpha_{1}^{-}=-(1 / \alpha+1)$ and $\alpha_{2}^{-}=-2$. Further, $Y_{i}=y_{n}-h / \alpha_{i}^{-}$and represents the intersections of the paths of integration for the kernel elements $P_{i j}^{-+}$with time $s_{k-1}$ (Figure 7). The corrector-iteration formulae for a computational molecule of the uniform mesh is

$$
\begin{aligned}
& \left(P_{i j, n k}^{++}\right)^{(p+1)}=P_{i j}^{++}\left(y_{n-1}, x, s_{k-2+i}\right)+\frac{h}{2 \alpha_{i}^{+}}\left(\mathcal{J}_{i j}^{+}\left(y_{n-1}, s_{k-2+i}\right)+\mathcal{J}_{i j}^{\prime+}\left(y_{n}, s_{k}\right)\right) \\
& \left(P_{i j, n k}^{-+}\right)^{(p+1)}=P_{i j}^{-+}\left(Y_{i}, x, s_{k-1}\right)+\frac{h}{2 \alpha_{i}^{-}}\left(\mathcal{J}_{i j}^{-}\left(Y_{i}, s_{k-1}\right)+\mathcal{J}_{i j}^{\prime-}\left(y_{n}, s_{k}\right)\right)
\end{aligned}
$$

In these formulas, both $\mathcal{J}_{i j}^{\prime+}\left(y_{n}, s_{k}\right)$ and $\mathcal{J}_{i j}^{\prime-}\left(y_{n}, s_{k}\right)$ involve $\left(P_{i j, n k}^{ \pm+}\right)^{(p)}$.

The superscript $p=0,1,2, \ldots$ enumerates the iteration sequence for $P_{i j, n k}^{ \pm+}$with both $n$ and $k$ fixed. The iteration for the uniform mesh is conducted for $n=0,1, \ldots, N$ with $k$ fix. The points of influence on the point of calculation, $\left(y_{n}, s_{k}\right)$, belong to the set $\left\{\left(y_{n-1}, s_{k}\right)\right\} \cup\left\{\left(y_{q}, s_{r}\right): q=\right.$ $n-1, n, n+1 ; 0 \leq r \leq k-1\}$.

The temporal convolutions of the functionals stated in (9.2) are approximated by the trapezoidal quadrature rule using the uniform time step $h$. The discretized versions of $\mathcal{J}^{ \pm}$are each subdivided into two parts. One that depends on $\mathbf{P}^{ \pm+}\left(y, s_{p}\right)$ for past time, $0<p \leq k-1$, and one extracted part that depends on $\mathbf{P}^{ \pm+}\left(y, s_{k}\right)$, respectively. Note that $P_{i j}^{++}=0$ at the left boundary and therefore only the iterations for $P_{i j}^{-+}$have to be conducted at $x=0$. Also, at the right boundary $P_{i j}^{-+}=0$ and only the elements $P_{i j}^{++}$have to be iterated for at $x=1$. At all interior points the kernel elements are simultaneously iterated for.

Algorithm 1 The scheme for computation of the propagator kernels.

1. Determine the kernel for all mesh nodes at $s_{1}$ : The initial conditions $P_{i j}^{++}(x, 0)$ are sequen- 


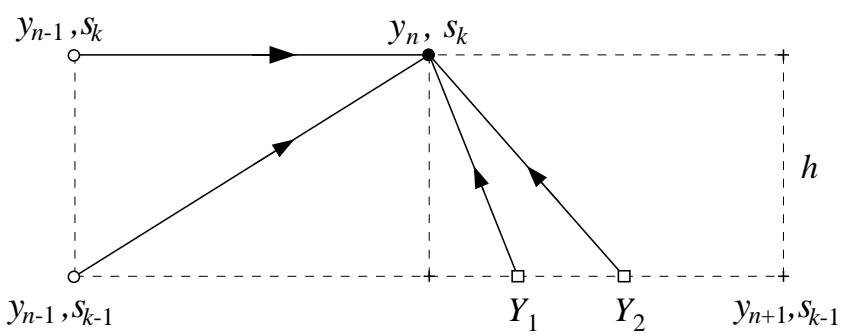

Figure 7: The computational molecule.

tially inserted into the predictor-corrector procedure, (9.3) and (9.4). Thus the discrete set $\left\{P_{i j, n 1}^{ \pm+}\right\}_{n=0}^{N}$ is obtained.

2. Determine the kernel elements for all off-node interpolation points at $s_{1}$ : The $\operatorname{set}\left\{P_{i j, n 1}^{ \pm+}\right\}_{n=0}^{N}$ is linearly interpolated, from the uniform mesh, to retrieve $P_{i j}^{ \pm+}\left(Y_{i}, s_{1}\right)$ for all $Y_{i} \in[x, 1]$. In performing the linear interpolation, knowledge of the intersections of the discontinuity characteristics with the present time step is used to ensure that no interpolation is performed across such a characteristic curve. If a point of interpolation should fall in between a node and a jump discontinuity, then the value at the node is used (zeroth order interpolation).

3. Repeat the procedure: The iteration procedure is subsequently repeated to obtain the set $\left\{P_{i j, n 2}^{ \pm+}\right\}_{n=0}^{N}$, which is in turn interpolated to give $P_{i j}^{ \pm+}\left(Y_{i}, s_{2}\right)$ for all $Y_{i} \in[x, 1]$. Steps 1-3 of this scheme are then applied repeatedly until a pre-set final time has been reached.

\section{Numerical example}

The numerical example concerns a homogeneous beam on a viscoelastic layer. In the example the relevant material parameters are chosen to match those of a beam of square cross section. The Poisson ratio is set to $\nu=0.3$ and the shear coefficient $k^{\prime}$ is chosen in accordance with [20]; $k^{\prime}=10(1+\nu) /(12+11 \nu)=0.85$. The corresponding velocity thus becomes $\alpha=0.57$. Further, the length of the viscoelastic suspension need not be explicitly stated, since only the ratio of the suspension-length $d$ to the width $a$ of the cross section enters into the equation. This ratio is set to $d / a=0.30$ in order to make comparisons with the results from [5].

Concerning the viscoelastic layer, the viscous damping is modelled by exponential memory kernels of the form $K_{i}(s)=-k_{i} e^{-s}$, where $k_{i}$ are the spring constants. In similarity with [5] the spring constants are set to $k_{1}=k_{2}=20.0$ in all the numerical examples. The discretization time 


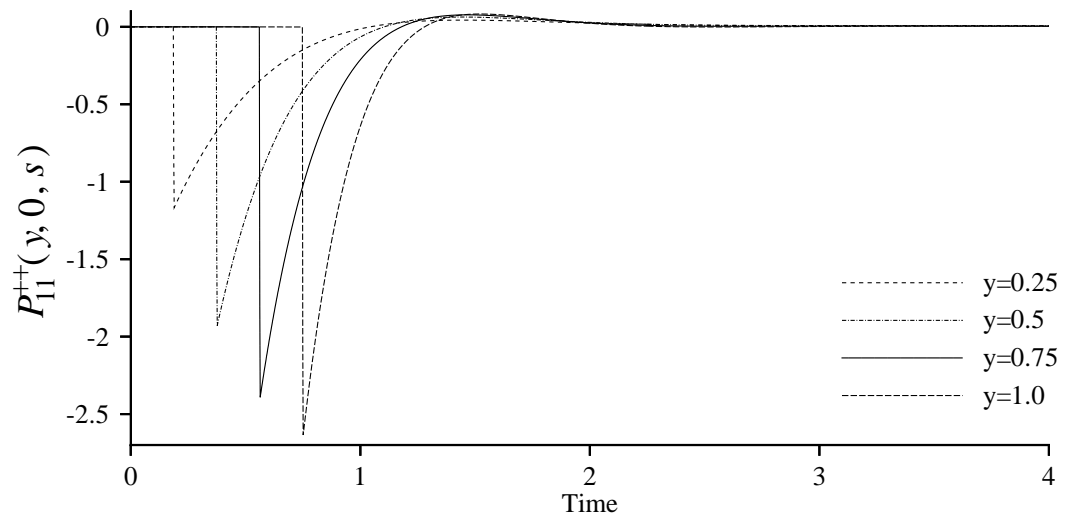

(a) $P_{11}^{++}(y, 0, s)$ for $y=0.25,0.5,0.75$ and 1.0 .

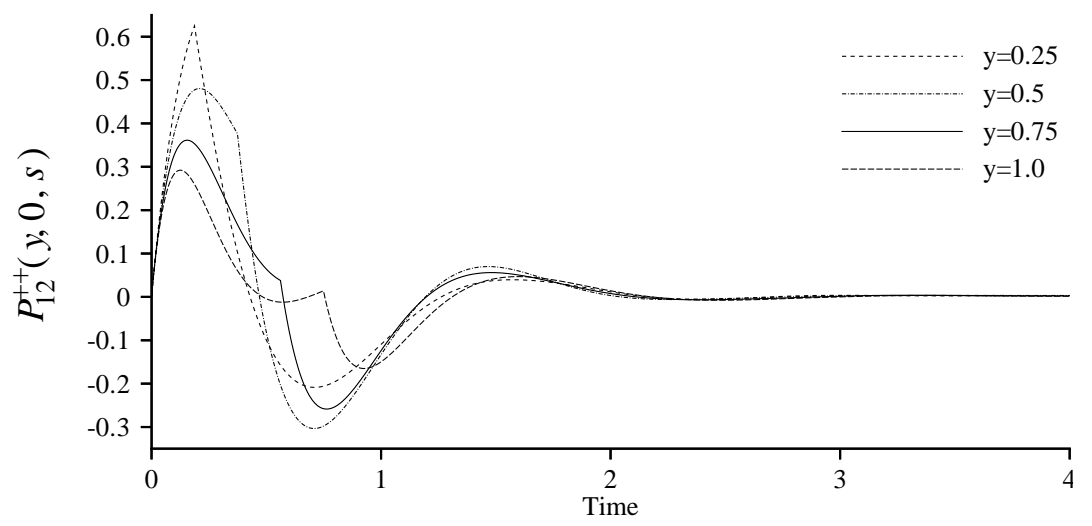

(b) $P_{12}^{++}(y, 0, s)$ for $y=0.25,0.5,0.75$ and 1.0 .

Figure 8: The propagator kernels $P_{11}^{++}$and $P_{12}^{++}$for $x=0$. The time scale, $s$, is given in units of $d / c_{2}$. 


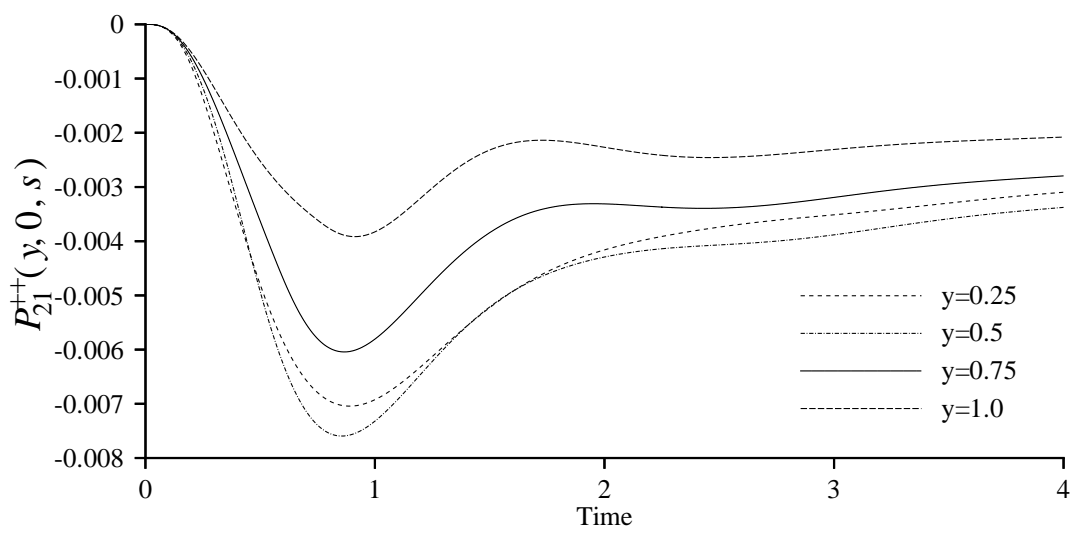

(a) $P_{21}^{++}(y, 0, s)$ for $y=0.25,0.5,0.75$ and 1.0.

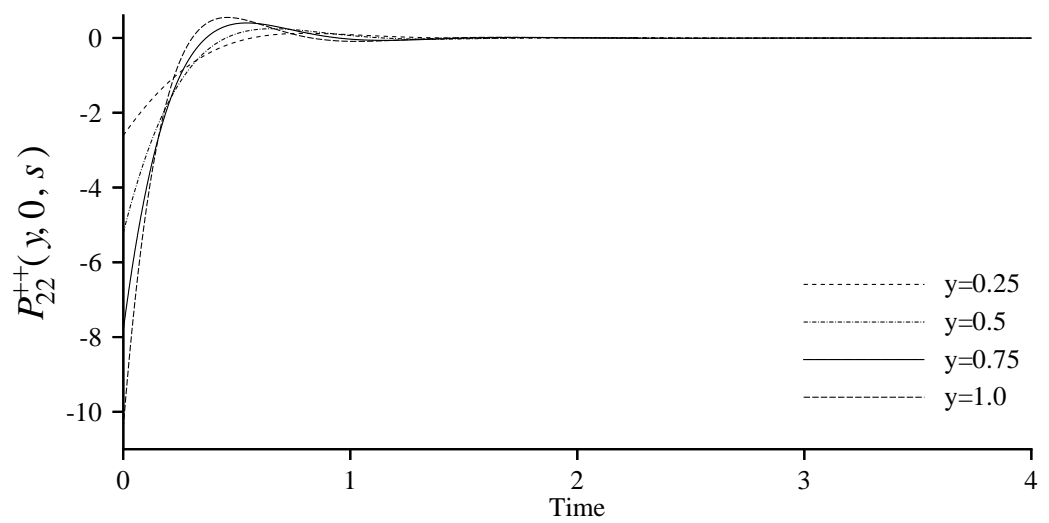

(b) $P_{22}^{++}(y, 0, s)$ for $y=0.25,0.5,0.75$ and 1.0 .

Figure 9: The propagator kernel $P_{21}^{++}$and $P_{22}^{++}$for $x=0$. The time scale, $s$, is given in units of $d / c_{2}$. 
step is $h=0.005$. Further, since the beam is homogeneous, the scaling function $\mu$ according to (8.9) is constant.

Figures 8 and 9 present the numerical results for the elements of the kernels $\mathbf{P}^{++}$for a series of $y$. The corresponding results for the elements of $\mathbf{P}^{-+}$are shown in Figures 10 and 11 . In all of these examples $x=0$, so in conformity with what is stated in Section 7.1 the functions presented are also the Green functions of the scattering region. Also, the elements of $\mathbf{P}^{-+}$corresponding to $y=0$ coincide with the elements of the imbedding reflection kernel, as was stated in Section 7.2. These results agree with those obtained by the numerical algorithm for the imbedding reflection equation, presented in [5]. Finally, note the jump discontinuities of $P_{11}^{++}$across $d_{1}^{+}$(Figure 8) and of $P_{22}^{++}$across $d_{0}^{+}$(Figure 9 ).

\section{Conclusion}

In this paper, the wave propagator method is applied in the study of of wave scattering on a viscoelastically restrained, inhomogeneous Timoshenko beam. The propagators are operators that map the incoming field at a fix cross section onto the scattered fields at an arbitrary position. The aim of the work is to present equations for the propagator kernels together with expressions for their jump discontinuities. In addition, certain symmetry properties are obtained for the coupling matrices of an inhomogeneous beam as well as for a homogeneous beam subjected to viscoelastic damping. The behaviour of the propagator kernels is presented in a numerical example concerning the viscoelastically restrained beam. The imbedding approach and the Green function technique are related to the propagator formalism. Especially, the propagator kernel $\mathbf{P}^{-+}(y, x, s)$ is equal to the imbedding reflection kernel in the limit $(y, x) \rightarrow(0,0)$. This fact is used to check the agreement of the numerical results for the propagators with the results for the corresponding reflection kernel obtained by a previously developed algorithm.

A future extension of this work is to study the wave propagation in periodic structures. As an example, a railroad track may be modelled by a homogeneous beam resting on a periodic layer. It is likely that the propagators obtained for one period may be used in an efficient way to develop propagators for an arbitrary number of periods, see [7]. Also, it would be of interest to study the propagation of waves on a homogeneous beam on an elastic foundation with spatially varying spring constants. The propagator method may be used to include the influence of distributed piezoelectric sensor and actuator layers on the beam, which has applications in structural control technologies. 


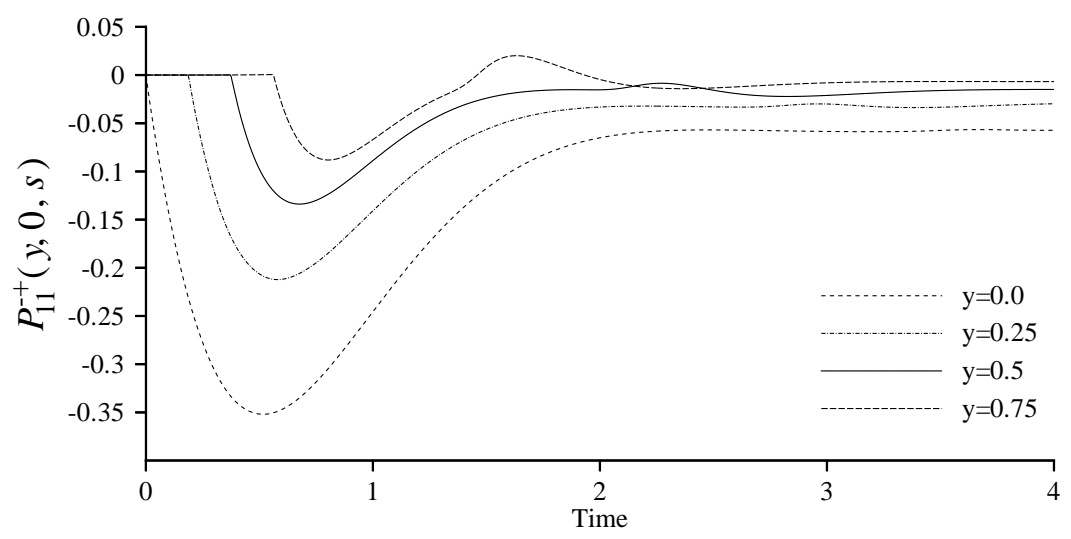

(a) $P_{11}^{-+}(y, 0, s)$ for $y=0.0,0.25,0.5$ and 0.75 .

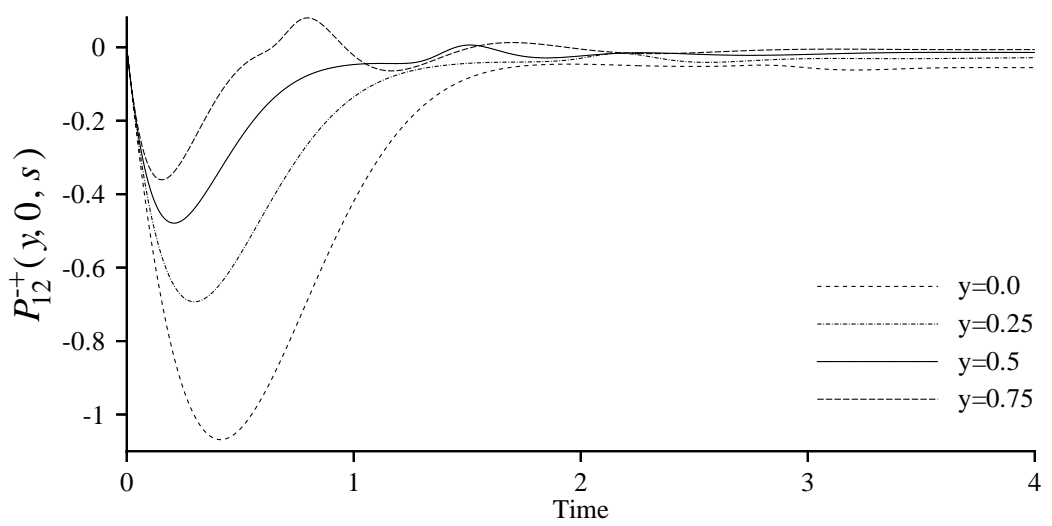

(b) $P_{12}^{-+}(y, 0, s)$ for $y=0.0,0.25,0.5$ and 0.75 .

Figure 10: The propagator kernels $P_{11}^{-+}$and $P_{12}^{-+}$for $x=0$. The time scale, $s$, is given in units of $d / c_{2}$. 


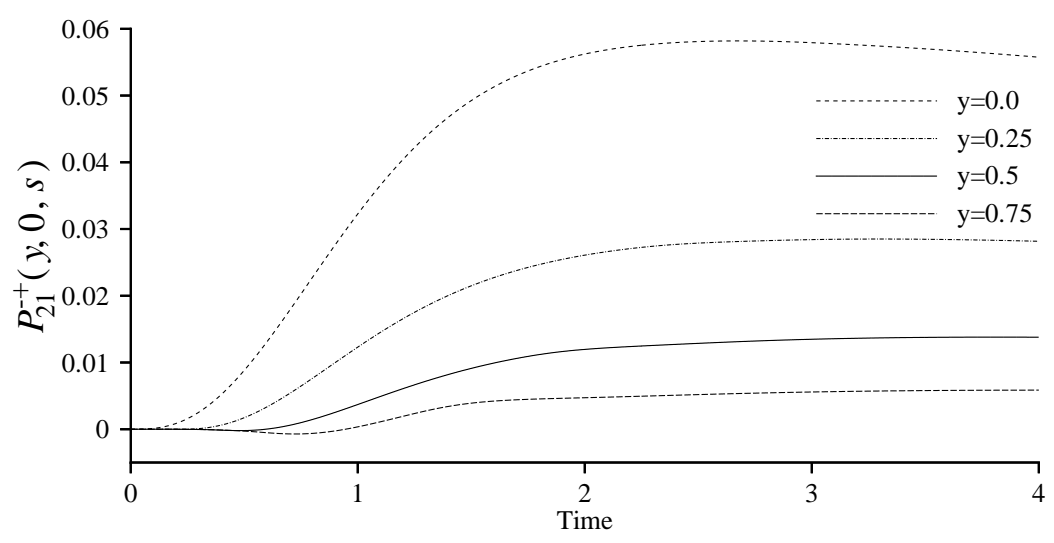

(a) $P_{21}^{-+}(y, 0, s)$ for $y=0.0,0.25,0.5$ and 0.75 .

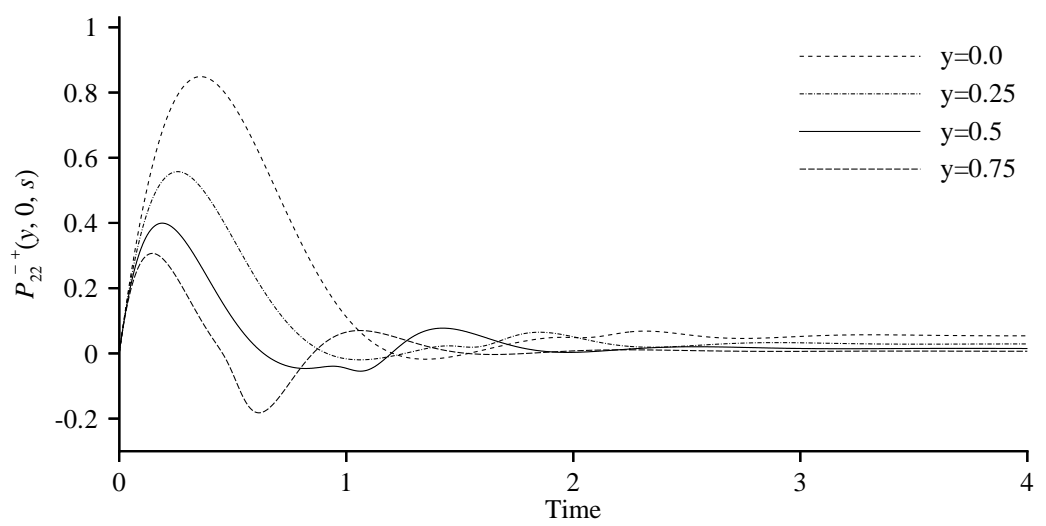

(b) $P_{22}^{-+}(y, 0, s)$ for $y=0.0,0.25,0.5$ and 0.75 .

Figure 11: The propagator kernels $P_{21}^{-+}$and $P_{22}^{-+}$for $x=0$. The time scale, $s$, is given in units of $d / c_{2}$. 


\section{Acknowledgement}

The authors wish to thank Professor Peter Olsson at the Department of Mechanics for help and inspiring support. The work in this paper is partially supported by a grant from The Swedish Research Council for Engineering Sciences (TFR) and this is gratefully acknowledged.

\section{A Wave propagators for sources located in $z>1$}

This appendix treats an analogous scattering problem to the one presented above. The only difference being that all sources are now present in the region $z>1$ and generate fields that propagate in the negative $z$-direction (Figure 12). The aim is to study the corresponding propagators and to

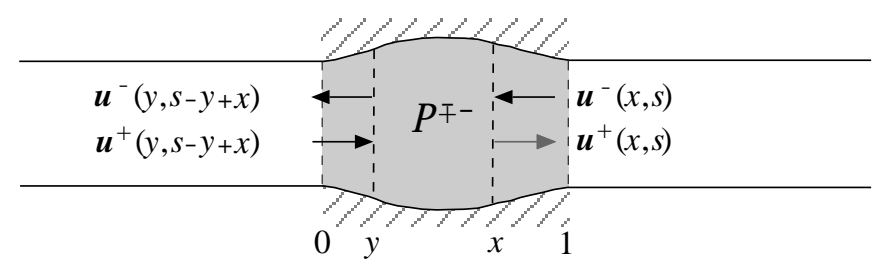

Figure 12: Scattering from sources in $z>1$.

derive equations for the propagator kernels. Since the procedure is similar to that of Section 4,5 and 6 , the analysis is kept to a minimum. The definitions of the wave propagators are analogous to $(4.1)$,

$$
\boldsymbol{u}^{\mp}(y, s-y+x)=\mathcal{P}^{\mp-}(y, x) \boldsymbol{u}^{-}(x, s) .
$$

Consequently, interchanging the plus and minus signs, the composition rules (4.2)-(4.3) apply here. The explicit representations of the propagators follow from (4.6)

$$
\begin{aligned}
& \boldsymbol{u}^{-}(y, s-y+x)=\boldsymbol{t}^{-}(y, x) \mathcal{S}(y, x) \boldsymbol{u}^{-}(x, s)+\left(\mathbf{P}^{--}(y, x, \cdot) * \boldsymbol{u}^{-}(x, \cdot)\right)(s), \\
& \boldsymbol{u}^{+}(y, s-y+x)=\left(\mathbf{P}^{+-}(y, x, \cdot) * \boldsymbol{u}^{-}(x, \cdot)\right)(s) .
\end{aligned}
$$

Here, the wave front matrix is $\boldsymbol{t}^{-}(y, x)=\operatorname{diag}\left(t_{1}^{-}(y, x), t_{2}^{-}(y, x)\right)$ where $t_{1}^{-}(y, x)=e^{\int_{x}^{y} N_{2211}\left(y^{\prime}\right) d y^{\prime}}$ and $t_{2}^{-}(y, x)=e^{\int_{x}^{y} N_{2222}\left(y^{\prime}\right) d y^{\prime}}$.

Using the dynamics (3.7), the equations for the propagator kernels that corresponds to (5.2) 
and (5.3) are

$$
\begin{aligned}
& \partial_{y} \mathbf{P}^{--}-\left(\mathbf{C}^{-1}-\mathbf{I}\right) \partial_{s} \mathbf{P}^{--}=\left(\mathcal{S}\left(\mathbf{M}_{22}^{T}+\mathbf{F}\right)\right)^{T} \boldsymbol{t}^{-} \\
& +\mathbf{N}_{22} \mathbf{P}^{--}+\mathbf{N}_{21} \mathbf{P}^{+-}+\left(\mathbf{M}_{22}+\mathbf{F}\right) * \mathbf{P}^{--}+\mathbf{M}_{21} * \mathbf{P}^{+-} \\
& \partial_{y} \mathbf{P}^{+-}+\left(\mathbf{C}^{-1}+\mathbf{I}\right) \partial_{s} \mathbf{P}^{+-}=\left(\mathcal{S} \mathbf{M}_{12}^{T}\right)^{T} \boldsymbol{t}^{-} \\
& +\mathbf{N}_{12} \mathbf{P}^{--}+\mathbf{N}_{11} \mathbf{P}^{+-}+\mathbf{M}_{12} * \mathbf{P}^{--}+\left(\mathbf{M}_{11}-\mathbf{F}\right) * \mathbf{P}^{+-}
\end{aligned}
$$

The equations for the discontinuities become

$$
\begin{aligned}
& \mathbf{N}_{22}^{0} \boldsymbol{t}^{-} \mathcal{S} \boldsymbol{u}^{-}-\sum_{i=0}^{n}\left(\mathbf{C}^{-1}-\mathbf{I}-\partial_{y} d_{i}^{-} \mathbf{I}\right)\left[\mathbf{P}^{--}\right]_{i} \boldsymbol{u}^{-}\left(x, s-d_{i}^{-}\right)=0, \\
& \mathbf{N}_{12} \boldsymbol{t}^{-} \mathcal{S} \boldsymbol{u}^{-}-\sum_{i=0}^{n}\left(\mathbf{C}^{-1}+\mathbf{I}+\partial_{y} d_{i}^{-} \mathbf{I}\right)\left[\mathbf{P}^{+-}\right]_{i} \boldsymbol{u}^{-}\left(x, s-d_{i}^{-}\right)=0
\end{aligned}
$$

where $\mathbf{N}_{22}^{0}=\mathbf{N}_{22}-\operatorname{diag}\left(N_{2211}, N_{2222}\right)$. For a homogeneous beam on a viscoelastic foundation, it is clear from (5.2), (5.3), (A.1) and (A.2) that $\mathbf{P}^{ \pm+}=\mathbf{P}^{\mp-}$ up to the time when the first jump discontinuity due to reflection at an end is present.

The jump discontinuities may exist across

$$
\begin{aligned}
& d_{0}^{-}(y, x)=0, \quad d_{1}^{-}(y, x)=\sigma(x, y)+y-x, \quad d_{2}^{-}(y, x)=2 y \\
& d_{3}^{-}(y, x)=\sigma(y, 0)+y, \quad d_{4}^{-}(y, x)=\sigma(x, 0)+2 y-x, \quad d_{5}^{-}(y, x)=\sigma(y, 0)+\sigma(x, 0)+y-x . \\
& \mathbf{P}^{--} \text {have jumps across } d_{0}^{-} \text {and } d_{1}^{-} \text {according to } \\
& {\left[P_{12}^{--}\right]_{0}=\frac{-\alpha}{1-\alpha} N_{2212} t_{2}^{-}, \quad\left[P_{21}^{--}\right]_{0}=\frac{-\alpha(x)}{1-\alpha(x)} N_{2221}(x) t_{2}^{-}, \quad\left[P_{22}^{--}\right]_{0}=t_{2}^{-} \int_{y}^{x} f_{22}^{-}\left(y^{\prime}\right) d y^{\prime},} \\
& {\left[P_{11}^{--}\right]_{1}=t_{1}^{-} \int_{y}^{x} f_{11}^{-}\left(y^{\prime}\right) d y^{\prime}, \quad\left[P_{12}^{--}\right]_{1}=\frac{\alpha(x)}{1-\alpha(x)} N_{2212}(x) t_{1}^{-}, \quad\left[P_{21}^{--}\right]_{1}=\frac{\alpha}{1-\alpha} N_{2221} t_{1}^{-},}
\end{aligned}
$$

where

$$
\begin{aligned}
& f_{11}^{-}=-M_{2211}\left(y, 0^{+}\right)-F_{1}\left(y, 0^{+}\right)-\frac{\alpha}{2} N_{1211} N_{2111}-\frac{\alpha}{1-\alpha} N_{2212} N_{2221}-\frac{\alpha}{1+\alpha} N_{1221} N_{2112} \\
& f_{22}^{-}=-M_{2222}\left(y, 0^{+}\right)-F_{2}\left(y, 0^{+}\right)-\frac{1}{2} N_{2122} N_{1222}+\frac{\alpha}{1-\alpha} N_{2212} N_{2221}-\frac{\alpha}{1+\alpha} N_{2121} N_{1212}
\end{aligned}
$$


The discontinuities of $\mathbf{P}^{+-}$are given as

$\left[P_{11}^{+-}\right]_{1}=\frac{\alpha}{2} N_{1211} t_{1}^{-}, \quad\left[P_{12}^{+-}\right]_{0}=\frac{\alpha}{1+\alpha} N_{1212} t_{2}^{-}, \quad\left[P_{21}^{+-}\right]_{1}=\frac{\alpha}{1+\alpha} N_{1221} t_{1}^{-}, \quad\left[P_{22}^{+-}\right]_{0}=\frac{1}{2} N_{1222} t_{2}^{-}$,

together with

$$
\begin{array}{ll}
{\left[P_{11}^{+-}\right]_{5}=-\frac{\alpha(0)}{2} N_{1211}(0) t_{1}^{-}(0, x) t_{1}^{+}(y, 0),} & {\left[P_{12}^{+-}\right]_{3}=-\frac{\alpha(0)}{1+\alpha(0)} N_{1212}(0) t_{2}^{-}(0, x) t_{1}^{+}(y, 0),} \\
{\left[P_{21}^{+-}\right]_{4}=-\frac{\alpha(0)}{1+\alpha(0)} N_{1221}(0) t_{1}^{-}(0, x) t_{2}^{+}(y, 0),} & {\left[P_{22}^{+-}\right]_{2}=-\frac{1}{2} N_{1222}(0) t_{2}^{-}(0, x) t_{2}^{+}(y, 0) .}
\end{array}
$$

\section{References}

[1] P. Olsson and G. Kristensson. Wave splitting of the Timoshenko beam equation in the time domain. Zeitschrift für andgewandte Mathematik und Physik, 45:866-881, 1994.

[2] P.D. Folkow, G. Kristensson, and P. Olsson. Time domain Green functions for the homogeneous Timoshenko beam. Q. Jl. Mech. appl. Math., 51(1):125-141, 1998.

[3] D.V.J. Billger and P.D. Folkow. The imbedding equations for the Timoshenko beam. Journal of Sound and Vibration, 209(4):609-634, 1998.

[4] D.V.J. Billger. Scattering on the Timoshenko beam- direct and inverse problems in the time domain. PhD thesis, Chalmers University of Technology, 1998.

[5] D.V.J. Billger and D.J.N. Wall. A time domain algorithm for the reflection of waves on a viscoelastically supported Timoshenko beam. Q. Jl. Mech. appl. Math., 52(2):211-236, 1999.

[6] P.D. Folkow. Time domain inversion of a viscoelastically restrained Timoshenko beam. Inverse Problems, 15(2):551-562, 1999.

[7] A. Karlsson and R. Stewart. Wave propagators for transient waves in periodic media. J. Opt. Soc. Am. A, 12(7):1513-1521, 1995.

[8] P. Bernekorn, A. Karlsson, and G. Kristensson. Propagation of transient electromagnetic waves in inhomogeneous and dispersive waveguides. J. Electro. Waves Applic., 10(9):1263$1286,1996$. 
[9] J. Fridén and G. Kristensson. Transient external 3D excitation of a dispersive and anisotropic slab. Inverse Problems, 13(3):691-709, 1997.

[10] A. Karlsson. Wave propagators for transient waves in one-dimensional media. Wave Motion, 24(1):85-99, 1996.

[11] A. Karlsson and K Kreider. Transient electromagnetic wave propagation in transverse periodic media. Wave Motion, 23:259-277, 1996.

[12] S. Rikte. The theory of the propagation of TEM-pulses in dispersive bi-isotropic slabs. Wave Motion, 29(1):1-21, 1999.

[13] A. Karlsson and S. Rikte. Time-domain theory of forerunners. J. Opt. Soc. Am. A, 15(2):487$502,1998$.

[14] S. He and S. Ström. Time-domain wave splitting and propagation in dispersive media. $J$. Opt. Soc. Am. A, 13(11):2200-2207, 1996.

[15] R. Hellberg. A Time-Domain Method for the Design of Non-Reflecting Media. PhD thesis, Royal Institute of Technology, 1997.

[16] M. Romeo. Wave splitting in linear viscoelasticity. Eur. J. Mech., A/Solids, 18:539-553, 1999.

[17] I. Egorov and S. Rikte. Forerunners in bigyrotropic materials. J. Opt. Soc. Am. A, 15(9):23912403, 1998.

[18] S. Rikte. Sommerfeld's forerunner in stratified isotropic and bi-isotropic media. Technical Report LUTEDX/(TEAT-7036)/1-26/(1994), Lund Institute of Technology, Department of Electromagnetic Theory, P.O. Box 118, S-211 00 Lund, Sweden, 1994.

[19] S.P. Timoshenko. On the correction for shear of the differential equation for transverse vibrations of prismatic bars. Phil. Mag., XLI:744-746, 1921. Reprinted in The Collected Papers of Stephen P. Timoshenko, McGraw-Hill, London 1953.

[20] G.R. Cowper. The shear coefficient in Timoshenko's beam theory. J. Appl. Mech., 33:335-340, 1966. 